\title{
Cosmic microwave background and parametric resonance in reheating
}

\author{
A. B. Henriques \\ Departamento de Fisica/CENTRA,Instituto Superior Tecnico, 1096 Lisbon, Portugal \\ R. G. Moorhouse \\ Department of Physics and Astronomy, University of Glasgow, Glasgow G12 8QQ, U.K.
}

(October 26, 2018)

\begin{abstract}
The variation of the perturbative 3 -curvature parameter, $\zeta$, is investigated in the period of reheating after inflation. The two-field model used has the inflaton, with an extra scalar field coupled to it, and non-linear effects of both fields are included as well as a slow decay mechanism into the hydrodynamic fluid of the radiation era. Changes in $\zeta$ occur and persist into the succeeding cosmic eras to influence the generation of the cosmic microwave background fluctuations. With appropriate coupling the extra field rises from almost zero at the end of inflation to parametric resonance which is important in giving ultimate changes to $\zeta$. Inflaton particles are always produced in important quantities and their interaction with the extra field may limit its parametric resonance. When the field coupling is such that parametric resonance is small or absent then non-linear effects of the produced inflaton particles by themselves also give ultimate changes in $\zeta$, though these are small.
\end{abstract}

\section{INTRODUCTION}

In the cosmic inflationary scenario parametric excitation of extra fields may mediate the transition of the inflaton field into a cosmic radiation era of a gas of relativistic particles [1 9 9. There has been discussion of the possible role of non-linear effects in such transitions [10 14]; depending on their magnitude such effects may be important in connecting the quantum excitations of the inflationary era inflaton field to the cosmic microwave background radiation fluctuations (CMBRF). When non-linear effects are not important this connection has often been made rather directly, for example by using the time-invariant properties of a parameter $\zeta$, associated with spatial curvature perturbations [15 17], for wavelengths appropriate to the CMBRF. To study such non-linear reheating effects on the CMBRF we calculate using the simplest theory as a test-bed with just one additional scalar field subject to parametric resonance [10,11].

Thus we have a model with an inflaton field $\varphi=\varphi_{0}+\varphi_{1}$, where $\varphi_{0}$ denotes the 'classical' part of the inflaton field and $\varphi_{1}$ the part arising from primordial quantum perturbations 
of $\varphi$. We use the perturbed FRW metric in longitudinal gauge which appears with the perturbing fields $\psi$ and $\Phi$ as

$$
d s^{2}=a(\tau)^{2}(1+2 \Phi) d \tau^{2}-a(\tau)^{2}(1-2 \psi) \delta_{i j} d x^{i} d x^{j} .
$$

In the usual case of absence of space-space off-diagonal elements of the stress-energy tensor $\psi$ and $\Phi$ are equal. The one additional field $\chi$ is taken to have the well-used interaction term $\frac{1}{2} g^{2} \varphi^{2} \chi^{2}$ [1 5]. Persuasive arguments have been given [12 14 that $\chi$ is strongly suppressed before reheating begins. It is then reasonable to treat $\chi$ as a field arising from its own quantum fluctuations without any primordial classical part. We thus have 3 initial quantum fields $\psi, \varphi_{1}$ and $\chi$. The equations of motion imply that in the canonical decomposition of quantum fields into momentum component creation and annihilation operators, the operators for $\psi$ and $\varphi_{1}$ are the same and those for $\chi$ are independent.

As shown by Polarski and Starobinsky 18 the quantum fields decohere into classical stochastic fields which nevertheless keep important properties of the initial quantum fields; for instance the ensemble averaging of the products of stochastic fields give similar results to the vacuum expectation value of the corresponding quantum fields. Since we cannot realistically invoke a non-perturbative and exact quantum mechanical treatment such averages necessarily arise in our treatment of the equations of motion; for example from $\frac{1}{2} g^{2} \varphi^{2} \chi^{2}$ a term of the form $g^{2} \varphi_{0} \chi^{2}$ appears in the equation of motion of the classical homogeneous part $\varphi_{0}$ of the inflaton requiring for consistency the introduction of the ensemble average, $<\chi^{2}>$, of $\chi^{2}$.

This feature is important, for example in the case of the homogeneous Einstein equations where the contributions due to $\varphi_{1}$ and $\chi$ during the reheating period may become the dominant ones. Also in the non-homogneous Einstein equations $\varphi_{1}$ cannot be treated as a perturbation but should be viewed as the non-homogeneous part of the field $\varphi$. On the other hand the field $\psi(x, t)$ will be treated as a perturbation and in our numerical simulations checks will always be made to ensure that $\psi(x, t)$ is always much smaller than unity. The justification of this procedure has been discussed previously 10,11.

Our model also incorporates true heating through an elementary decay mechanism, with decay constants $\Gamma_{\varphi}$ and $\Gamma_{\chi}$, of the scalar fields into a hydrodynamical relativistic (radiation) fluid. The system thermalizes well with $\psi$ becoming approximately constant as expected from analytic calculations. We calculate, using well-known procedures, the cosmic microwave background fluctuations starting from the values of $\psi$ or $\zeta$ immediately after thermalization thus incorporating any changes that may have occured in the transition from inflation to the radiation era.

We shall see that we have to deal with two different regimes depending on the relative values of the particle or field energy densities attained in the transition. On the one hand we have the classical inflaton field, $\varphi_{0}$, whose density declines plus the inflaton particles field, $\varphi_{1}$, whose density increases; these densities become close to each other and stay so until they thermalize. On the other hand we have the $\chi$ density which at some time in the transition increases rapidly from almost zero to some non-negligable value. One regime, A, is that where the $\chi$ density stays below the other densities; the second regime, $\mathrm{B}$, which is induced by larger couplings than those of $\mathrm{A}$ is where the the $\chi$ density approaches rivalry with the others. Both regimes can be induced by a large number of different values of the parameters 
$g^{2}$, the coupling strength, and of $\Gamma_{\varphi}$ and $\Gamma_{\chi}$ which vary the rapidity of thermalization. In both regimes $\zeta$ changes but in $\mathrm{A}$, where the $\chi$ density remains comparatively modest, the change is constant and smaller while in B the change factor varies.

We know from analytic argument that when there are non-linear effects of the fields the usual theorems showing $\zeta$ to be constant fail, for example as shown in section IIIB below. Because of the complexity of the equations we do need numerical computation to verify and quantify the change in $\zeta$ and any importance it may have for calculation of the CMBRF. This paper attempts to do so for a particular model of preheating and reheating.

The paper has been organised as follows. In the next section we precisely define the model for the reheating period and give the resulting equations of motion. The initial conditions arising from the inflationary period are also given. We call attention to the fact that despite the initial extreme suppression and smallness of $\chi 12$ 14 this field still develops an important resonating behaviour. The theory can be formulated in different ways because of the redundancy of the many equations of motion and some small such changes have been made with respect to our previous papers [10,11] with the purpose of increasing the accuracy of the numerical computations. In section III we discuss the results of the computations and in section IV we make the connection with the cosmic microwave background fluctuations.

\section{THE REHEATING EQUATIONS}

\section{A. The Equations of Motion}

The scalar field part of the model is specified by the Lagrangian

$$
L=\int d^{4} x \sqrt{-g}\left[\frac{1}{2} \varphi^{, \alpha} \varphi_{, \alpha}+\frac{1}{2} \chi^{, \alpha} \chi_{, \alpha}-V(\varphi)-V(\chi)-V_{i n t}(\varphi, \chi)\right]
$$

where $\varphi=\varphi_{0}+\varphi_{1}$. . We take the field potentials during reheating to be

$$
V(\varphi)=\frac{1}{2} m^{2} \varphi^{2} ; V(\chi)=\frac{1}{2} M^{2} \chi^{2} ; V_{\text {int }}(\varphi, \chi)=\frac{1}{2} g^{2} \varphi^{2} \chi^{2} .
$$

The perturbed longitudinal gauge metric is given by Eq.(1), the dynamics of the evolution of the scale $a(\tau)$ by the Einstein homogeneous equation (12) below. It is characteristic of our treatment that we always include the metric perturbations. As in our physical system there are no space-space off-diagonal elements of the stress-energy tensor 円, $\Phi=\psi$ and we shall denote the metric perturbation field by $\psi$. A friction mechanism will also be adopted to simulate the decay to the thermal gas of relativistic particles dominating subsequent radiation era. These terms take the simple form $\Gamma_{\phi} \phi^{\prime 2}$ and $\Gamma_{\chi} \chi^{\prime 2}$ and they can be consistently

\footnotetext{
${ }^{1}$ The ensemble averaging of non linear terms, described below and more fully in ref. [10], suppresses any such terms which might otherwise be present in our non-linear theory. Corrections to the result $\Phi=\psi$ would be present in a full quantum mechanical treatment, but as stated in the introduction that is beyond the scope of the present work. Finelli and Khlebnikov [19] also find such corrections in a different context where the fields are classical rather than in a stochastic ensemble as here.
} 
introduced into the equations of motion in such a way that the Bianchi identities are satisfied [10].

The stress-energy tensor and the equations of motion involve the averages of bilinear products of stochastic fields. Using as an example $\varphi_{1}$ these are defined as follows:

$$
\left\langle\varphi_{1}(x, \tau)^{2}\right\rangle=(2 \pi)^{-3} \int d^{3} k \varphi_{\mathbf{k}} \varphi_{\mathbf{k}}^{*}
$$

where $\varphi_{\mathbf{k}}$ are the $\mathrm{k}$-mode functions entering in the expression

$$
\varphi_{1}(\mathbf{x}, \tau)=\int \frac{d^{3} k}{(2 \pi)^{\frac{3}{2}}}\left[e(\mathbf{k}) \varphi_{\mathbf{k}}(\tau) \exp (i \mathbf{k} \cdot \mathbf{x})+e^{*}(\mathbf{k}) \varphi_{\mathbf{k}}^{*}(\tau) \exp (-i \mathbf{k} \cdot \mathbf{x})\right]
$$

with $e(\mathbf{k})$ and $e^{*}(\mathbf{k})$ being time-independent $\delta$-correlated Gaussian variables [18] such that, where $\langle\ldots .$.$\rangle denotes the average,$

$$
\left\langle e(\mathbf{k}) e^{*}\left(\mathbf{k}^{\prime}\right)\right\rangle=\frac{1}{2} \delta^{3}\left(\mathbf{k}-\mathbf{k}^{\prime}\right)
$$

with averages of $e e$ and $e^{*} e^{*}$ products being zero.The field $\psi(\mathbf{x}, \tau)$ is expressed as Eq.(5) with the same gaussian variables but the mode functions being $\psi_{\mathbf{k}}(\tau)$. The stochastic gaussian variables of $\chi$ are independent. Thus, for example, $\left\langle\varphi_{1}(x, \tau) \chi(x, \tau)\right\rangle$ is zero while $\left\langle\varphi_{1}(x, \tau) \psi(x, \tau)\right\rangle$ and $\left\langle\chi(x, \tau)^{2}\right\rangle$ are non-zero [10.

The thermal gas of relativistic particles is described by the hydrodynamical variables $\rho$, the density, and $p$, the pressure, related by the equation of state $p=\frac{1}{3} \rho$. As with the inflaton field the density has two components

$$
\rho(\mathbf{x}, \tau)=\rho_{0}(\tau)+\rho_{1}(\mathbf{x}, \tau)
$$

being the homogeneous background (classical) density and the non-homogeneous quantum or stochastic part. As will be seen below the equations of motion imply that the stochastic variables of $\rho_{1}$ are the same as those of $\varphi_{1}$ and $\psi$. We assume that $\rho_{0}$ is zero at the beggining of reheating and only develops subsequently through the friction mechanism $\Gamma_{\phi} \phi^{2}$ and $\Gamma_{\chi} \chi^{\prime 2}$. In terms of consistency of the friction model the assumption that $\rho_{0}$ only becomes significant during the reheating is not unreasonable because the friction only becomes significant when the fields are oscillating rapidly.

From the Lagrangian and from the hydrodynamical variables $p$ and $\rho$ we define the energy-momentum tensor

$$
T_{\nu}^{\mu}=\varphi^{, \mu} \varphi_{, \nu}+\chi^{, \mu} \chi_{, \nu}-\left[\frac{1}{2} \varphi^{, \alpha} \varphi_{, \alpha}+\frac{1}{2} \chi^{, \alpha} \chi_{, \alpha}-V(\varphi)-V(\chi)-V_{i n t}-p\right] \delta_{\nu}^{\mu}+(\rho+p) u^{\mu} u_{\nu}
$$

where $u^{\mu}$ is the 4-velocity of the fluid.

The homogeneous part of the total density, $\rho_{T}(\mathbf{x}, \tau)$, is defined as $\rho_{0 T}(\tau)=<T_{0}^{0}>$, giving, after ensemble averaging,

$$
\rho_{0 T}(\tau)=\frac{1}{2 a^{2}}\left[\eta+a^{2} \bar{m}^{2}\left(\varphi_{0}^{2}+\left\langle\varphi_{1}^{2}\right\rangle\right)+a^{2} M^{2}\left\langle\chi^{2}\right\rangle+\left\langle\varphi_{1, i}^{2}\right\rangle+\left\langle\chi_{, i}^{2}\right\rangle-4 \varphi_{0}^{\prime}\left\langle\varphi_{1}^{\prime} \psi\right\rangle\right]+\rho_{0}
$$




$$
\begin{gathered}
\eta=\varphi_{0}^{\prime 2}+\left\langle\varphi_{1}^{\prime 2}\right\rangle+\left\langle\chi^{\prime 2}\right\rangle \\
\bar{m}^{2} \equiv m^{2}+g^{2}\left\langle\chi^{2}\right\rangle
\end{gathered}
$$

The definition of $p_{0 T}$ is slightly more subtle. Although we shall be using the Friedmann equation to calculate $a(\tau)$ :

$$
\left(a^{\prime} / a\right)^{2}=\frac{8 \pi G}{3} a^{2} \rho_{0 T}(\tau)
$$

we should ensure that this equation is indeed compatible with the second order (time-time) Einstein equation for $a^{\prime \prime} / a$ which is:

$$
a^{\prime \prime} / a-\left(a^{\prime} / a\right)^{2}=-\frac{4 \pi G}{3} a^{2}\left(\rho_{0 T}(\tau)+3 p_{0 T}(\tau)\right)
$$

Deriving Eq.(12) and comparing with Eq.(13) we find

$$
\begin{gathered}
p_{0 T}(\tau)=\frac{1}{2 a^{2}}\left[\eta_{-}-a^{2} \bar{m}^{2}\left(\varphi_{0}^{2}+\left\langle\varphi_{1}^{2}\right\rangle\right)-a^{2} M^{2}\left\langle\chi^{2}\right\rangle-4 \varphi_{0}^{\prime}\left\langle\varphi_{1}^{\prime} \psi\right\rangle\right]+ \\
\rho_{0} / 3-\left(a^{\prime} / a\right)^{-1}\left[2 a^{-2} \varphi_{0}^{\prime}\left\langle\varphi_{1} \psi^{\prime}\right\rangle+\frac{4}{3}\left\langle\rho_{1} \psi^{\prime}\right\rangle\right] . \\
\eta_{-}=\varphi_{0}^{\prime 2}+\left\langle\varphi_{1}^{\prime 2}\right\rangle+\left\langle\chi^{\prime 2}\right\rangle-\left\langle\varphi_{1, i}^{2}\right\rangle / 3-\left\langle\chi_{, i}^{2}\right\rangle / 3 .
\end{gathered}
$$

This differs, in the addition of the last term in square brackets, from the expression obtained by using $p_{0 T}(\tau) \delta_{j}^{i}=-\left\langle T_{j}^{i}\right\rangle$ which we have used previously [10, but it has the additional virtue of precisely maintaining the continuity (or 'energy conservation') equation. However in the equations of motion the expression for $p_{0 T}$ only occurs in the metric perturbation equation (Eq.(21) below) and in fact the addition of the last term seems to make little difference to the numerical results.

Besides the Friedmann equation there are equations of motion for the other homogeneous variables'], these being $\varphi_{0}$ and $\rho_{0}$ :

$$
\begin{gathered}
\varphi_{0}^{\prime \prime}+2\left(a^{\prime} / a\right) \varphi_{0}^{\prime}+a^{2} \bar{m}^{2} \varphi_{0}-4\left\langle\psi^{\prime} \varphi_{1}^{\prime}\right\rangle-4\left\langle\psi \nabla^{2} \varphi_{1}\right\rangle+2 a^{2} \bar{m}^{2}\left\langle\psi \varphi_{1}\right\rangle=-a \Gamma_{\phi}\left(\varphi_{0}^{\prime}+2\left\langle\psi \varphi_{1}^{\prime}\right\rangle\right) \\
\rho_{0}^{\prime}+4\left(a^{\prime} / a\right) \rho_{0}=a^{-1} \Gamma_{\varphi}\left(\varphi_{0}^{\prime 2}+\left\langle\varphi_{1}^{\prime 2}\right\rangle\right)+a^{-1} \Gamma_{\chi}\left\langle\chi^{\prime 2}\right\rangle-2 a^{-2} \varphi_{0}^{\prime}\left\langle\psi \nabla^{2} \varphi_{1}\right\rangle+4\left\langle\psi^{\prime} \rho_{1}\right\rangle
\end{gathered}
$$

There remain the spatially non-homogeneous equations which we write in the $k$-component form. These components are specified as the complex mode functions $\chi_{k}, \varphi_{k}, \psi_{k}$ and $\rho_{1 k}$. Their wave number dependence, given by the succeeding equations, is only on $k \equiv|\mathbf{k}|$. There are 4 equations which we take as those for $\chi_{k}, \varphi_{k}$ and the time-time and space-space

\footnotetext{
${ }^{2}$ Both equations can be obtained from the Bianchi identities as shown in Appendix A of ref. [10]
} 
Einstein equations for $\psi_{k}$. (These being satisfied the time derivative equation equation for $\rho_{1 k} \equiv 3 p_{1 k}$ is implicit through the Bianchi identities.)

$$
\chi_{k}^{\prime \prime}+2\left(a^{\prime} / a\right) \chi_{k}^{\prime}+\left(k^{2}+a^{2} \bar{M}^{2}\right) \chi_{k}=-a \Gamma_{\chi} \chi_{k}^{\prime}
$$

where $\bar{M}^{2}$ is a function of $\tau$ given by

$$
\begin{gathered}
\bar{M}^{2}(\tau)=M^{2}+g^{2} \varphi_{0}(\tau)^{2}+g^{2}\left\langle\varphi_{1}(\mathbf{x}, \tau)^{2}\right\rangle, \\
\varphi_{k}^{\prime \prime}+2\left(a^{\prime} / a\right) \varphi_{k}^{\prime}+\left(k^{2}+a^{2} \bar{m}^{2}\right) \varphi_{k}-4 \varphi_{0}^{\prime} \psi_{k}^{\prime}+2 a^{2} \bar{m}^{2} \varphi_{0} \psi_{k}=-a \Gamma_{\varphi}\left(\varphi_{k}^{\prime}+2 \varphi_{0}^{\prime} \psi_{k}\right),
\end{gathered}
$$

the equations actually holding for each separate value of $\mathbf{k}$; thus consistency of Eq.(20) justifies our previous statements that $\psi_{\mathbf{k}}$, the mode functions of the metric perturbation, should be associated with the the same stochastic variables (or quantum operators) as $\varphi_{\mathbf{k}}$.

The space-space Einstein equation for the metric perturbation can be written

$$
\psi_{k}^{\prime \prime}+3 \psi_{k}^{\prime}\left(a^{\prime} / a\right)+\psi_{k}\left(a^{\prime} / a\right)^{2}=4 \pi G a^{2} p_{T k}+\frac{8 \pi G}{3} a^{2}\left(\rho_{0 T}+p_{0 T}\right) \psi_{k}
$$

where $p_{T k}$ is the $k$-component of the non-homogeneous part of the total momentum:

$$
\begin{gathered}
p_{T k}=a^{-2}\left[-\eta_{+} \psi_{k}+\varphi_{0}^{\prime} \varphi_{k}^{\prime}-2 \varphi_{k}^{\prime}\left\langle\psi \varphi_{1}^{\prime}\right\rangle-a^{2} \bar{m}^{2} \varphi_{0} \varphi_{k}\right]+p_{1 k} \\
\eta_{+}=\varphi_{0}^{\prime 2}+\left\langle\varphi_{1}^{\prime 2}\right\rangle+\left\langle\chi^{\prime 2}\right\rangle+\left\langle\varphi_{1, i}^{2}\right\rangle / 3+\left\langle\chi_{, i}^{2}\right\rangle / 3
\end{gathered}
$$

The time-time Einstein equation is

$$
\begin{gathered}
3 \psi_{k}^{\prime}\left(a^{\prime} / a\right)+\psi_{k}\left(k^{2}+3\left(a^{\prime} / a\right)^{2}\right)=4 \pi G a^{2} \rho_{T k} . \\
\rho_{T k}=a^{-2}\left[-\left(\eta-\left\langle\varphi_{1, i} \varphi_{1, i}\right\rangle-\left\langle\chi_{, i} \chi_{, i}\right\rangle\right) \psi_{k}+\varphi_{0}^{\prime} \varphi_{k}^{\prime}-2 \varphi_{k}^{\prime}\left\langle\psi \varphi_{1}^{\prime}\right\rangle+a^{2} \bar{m}^{2} \varphi_{0} \varphi_{k}\right]+\rho_{1 k} .
\end{gathered}
$$

Rather than using the mode equation for $\rho_{1 k}^{\prime}$ to find $\rho_{1 k}$ we find it from Eqs. 24, 25) and then use the equation of state $p_{1 k}=\frac{1}{3} \rho_{1 k}$ to solve the $\psi$ equation (21,22). I

In addition there is the time-space Einstein equation which is not independent of the other two Einstein equations but which acts as an equation of constraint on the initial values where we use it to fix the value of $\psi^{\prime}$ at the beginning of reheating. It takes the form

$$
\psi_{k}^{\prime}+\left(a^{\prime} / a\right) \psi_{k}=4 \pi G \varphi_{0}^{\prime} \varphi_{k}
$$

\footnotetext{
${ }^{3}$ As are $\varphi_{k}$ and $\psi, \chi$ is complex and the real and imaginary parts are not constant multiples of each other for smaller occupation numbers of a mode

${ }^{4}$ The $\rho_{1 k}$ mode equation is still maintained; the Bianchi identities hold and imply the $\rho_{1 k}$ mode equation when the other equations of motion are explicitly fulfilled, as they are 10.
} 


\section{B. Initial conditions}

We need to have the values of $H$, the fields and their time-derivatives at the end of inflation to supply the initial conditions for the reheating equations of motion . We have chosen to use a specific inflationary model having the advantage that the solutions are analytically expressible. This is power-law inflation with an exponential potential: $V=$ $U \exp (-\lambda \varphi)$ where $U, \lambda$ are constants. This potential of the inflation era stands in place of the potential $V(\varphi)=\frac{1}{2} m^{2} \varphi^{2}$ of the reheating era but otherwise the Lagrangians are the same and in particular both have the interaction potential $V_{\text {int }}(\varphi, \chi)=\frac{1}{2} g^{2} \varphi^{2} \chi^{2}$. We have ensured the correct continuity of the potential by imposing the Lichnerowicz conditions [20], as well as using the equations of motion and constraint appropriate to each era at the boundary [10].

That the $\chi$-field be strongly suppressed in the inflation era, as referred to previously 12 14, is an essential feature in maintaining the analytic form of power-law inflation. The many efold increase of $a$ during the inflationary era indicates an exceedingly small value for $\chi_{k}$ at the beginning of reheat [12 14, which may be as little as $10^{-50}$ of its initial value. This is an important qualitative feature of our initial conditions. We have taken the initial value of $\chi$ to be $10^{-n} m_{\text {Planck }}^{-1 / 2}$ where $n$ is of the order of 30. (For such small mode functions in the beginning of reheat the transition to classical stochastic functions cannot yet be made and the complex formalism, which is applicable also to the quantum case [18,10 should be retained.

For power-law inflation the scale factor $a \propto\left(\tau_{i}-\tau\right)^{p}$ and we are free to choose $p$ by specifying $\lambda$ in the potential [10]. In the numerical results quoted we have chosen $p=-1.1$ ( $p=-1$ corresponds to exponential inflation).

Knowing the analytic solutions of the unperturbed equations enables the deduction of $\psi_{k}$ and $\varphi_{k}$ in the inflation era starting from the initial quantum oscillations [10]. These are

$$
\begin{gathered}
\psi_{k}=a^{-1} \sqrt{\frac{4 \pi G}{2 k}} \alpha \sqrt{|\gamma|}\left(\mu_{k}^{\prime}-\alpha \mu_{k}\right) / k^{2} \\
\varphi_{k}=a^{-1}(2 k)^{-1 / 2}\left[\mu_{k}+\alpha \gamma\left(\mu_{k}^{\prime}-\alpha \mu_{k}\right) / k^{2}\right]
\end{gathered}
$$

where $\gamma \equiv 1-\alpha^{\prime} / \alpha^{2}=(p+1) / p$ and

$$
\mu_{k}^{\prime \prime}+\left(k^{2}-a^{\prime \prime} / a\right) \mu_{k}=0
$$

Since $a^{\prime \prime} / a=\frac{p+1}{p}\left(\tau_{i}-\tau\right)^{-2}$, Eq. (29) can be expressed as a Bessels equation in terms of

$$
y \equiv k\left(\tau_{i}-\tau\right)=\frac{k}{a H}|p|
$$

and the solutions which satisfy the asymptotic conditions for a scalar field are

$$
\mu_{k}(y)=\sqrt{\frac{\pi y}{2}}\left(J_{\nu}(y)-i Y_{\nu}(y)\right) \exp \left[-i\left(\frac{1}{2} \nu \pi+\frac{1}{4} \pi\right)\right]
$$

where $\nu=1 / 2-p$. 
For $k / a \ll H$ (many times over fulfilled by the $k$ relevant to the CMBRF observations) $\mu_{k}(y) \propto y^{p} \propto k^{p}$ and if this $k$-dependence were to pass unaltered through the reheat era into the radiation era it would yield a power-law spectrum scaling as $k^{n-1}$ where $n$, the spectral index, is given by $n=2 p+3$.

From the conditions of continuity it follows that at the junction of inflation and reheat

$$
H=\sqrt{4 \pi G} m \varphi_{0} \sqrt{p /(2 p-1)}
$$

and this, using Eq.(30), determines the value of $y$ at the junction

\section{SOLVING THE REHEATING EQUATIONS}

One of the main aims of the present work is to see whether,and if so how far, parametric resonance of a second scalar influences the fluctuations of the CMBR.

An important parameter is $\zeta_{k}$ [15.16] which, for $k^{2} / a^{2} \ll H^{2}$, is equivalent to the curvature perturbation $\mathcal{R}_{k}$. $\zeta$ is defined in terms of the gauge-invariant metric perturbation $\Phi$, equal in our case to the longitudinal gauge perturbation $\psi$; in terms of this variable we have 17]

$$
\zeta_{k} \equiv \frac{2}{3}\left(H^{-1} \dot{\psi}_{k}+\psi_{k}\right) /(1+w)+\psi_{k} \approx-\mathcal{R}_{k}
$$

where $w=p_{0 T} / \rho_{0 T}$, the total (homogeneous) pressure to density ratio. If $k^{2} / a^{2} \ll H^{2}$ then $\zeta$ is constant during the radiation era and constant if there be only one scalar field, the inflaton, during inflation and also during reheating if the inflaton perturbation can be treated to first order. That is, as it is often expressed, it is constant from horizon exit during inflation to horizon re-entry around the beginning of the matter era. The question arises whether, with another scalar field having parametric resonance during reheating, can $\zeta_{k}$ exit reheating with a different value from that with which it enters [12, 23, 24, As we shall see, for values of $\mathrm{g} / \mathrm{m}$ bigger than about 5000 there is parametric resonance, with $\chi$ comparable to the other scalar densities, and $\zeta$ is larger than its initial valuef and also there can be contributions to this effect from higher order inflaton perturbations. Then, knowing $\zeta$ at the end of reheating (that is at the beginning of the radiation era, asessed as in the succeeding paragraph) we can use known methods to find some properties of the fluctuations and assess consequences of the change in $\zeta$ in reheat.

Another method [21,22] is to trace the metric perturbation $\psi_{k}$, for the appropriate very small values of $\mathrm{k}$, from the end of reheating to the matter era where the Sachs-Wolf effect comes into play. Once the system thermalizes, with the gas of relativistic particles obeying an equation of state of the form $p=\rho / 3$, we can check that as the reheating era tends to the beginning of the radiation era then $\psi_{k}=b 1+b 2 / a^{3}, a(\tau) \approx \tau$, is a solution of Eqs. (21),(24), for $k^{2} \approx 0$, where the constant b1 has to be determined from the computations.

\footnotetext{
${ }^{5}$ This is a correction to some numerical values given in our previous work. Due to a misprint in the computing code some decreasing values of $\zeta$ for large couplings were, wrongly, found.
} 
The attainment of constant value for $\psi_{k \approx 0}$ and $\rho a^{4}$ will be taken as indicating the beginning of the radiation era; $\psi$ can then be traced throughout the radiation era from its analytic form which holds therein.

In this paper we evaluate the power spectrum of the fluctuations and find the quadrupole moment (which can give the COBE normalization) using the first method above.

In contrast to our results given below, the work of refs. [12] finds very small effects of $\zeta$ variation by expressing $\dot{\zeta}$ in terms of the non-adiabatic pressure variation. This comes using the reasonable physical assumption - based on the approximate validity of the RobertsonWalker metric - that after smoothing on a cosmological scale below the ones of interest the spatially inhomogeneous variables such as $\rho_{k}, p_{k}$ can be treated as perturbations. There is no evident agreement between that expression for $\dot{\zeta}$ and Eq. (34) below which uses no assumptions on $\rho_{k}, p_{k}$ being perturbative. However, as we discuss in sections I and V, in our work we do consider the variation $\psi$ from the Robertson-Walker metric to be perturbative.

\section{A. Small coupling constant, $\mathrm{g} / \mathrm{m}$}

Having derived in Sec. IIB the expressions allowing the magnitude of the relevant modes at the beginning of reheat to be calculated, thus fixing the input of $\psi, \varphi_{1}$ and $\chi$ to the reheat era, we may proceed with the numerical integration of the reheating equations of Sec.ПIA, taking the integration up to a point where we may consider the system to be well thermalized. The output of this numerical development will then be used in Sec.IV.

Unless otherwise stated, the results are for $p=-1.1, M / m=0.02, \phi_{0}=.3 m_{\text {Planck }}$ at the beginning of reheat and the inflaton mass $m=10^{-7} m_{\text {Planck }}\left(m_{\text {Planck }}=G^{-1 / 2}\right)$. The values for the coupling constant $g$, in $\frac{1}{2} g^{2} \varphi^{2} \chi^{2}$, will be quoted in terms of $g / m$.

The frictional decay constants $\Gamma_{\chi}, \Gamma_{\varphi}$ have been investigated in the range $0.1>\Gamma_{\chi} / m>0$ and $0.005>\Gamma_{\varphi} / m>0$, larger values tending to suppress the resonance. However the results presented here will be for small values of the order of $10^{-4}$. The reason is that then the parametric resonance has had time to reach its full effect before the $\varphi_{0}, \varphi_{1}$ and $\chi$ decay. For larger decay constants the transition to the radiation era occurs when $\zeta$ is still fluctuating very strongly leading to more random $\zeta$ values in the radiation era. It is significant that without this physical assumption the theory would be very randomly dependent on the precise magnitude of the coupling.

In this subsection we consider values of $g / m<4000$ and some typical results are given in Table I. The main conclusion seems to be the fact that the end of reheating values of our variables $\psi_{k \approx 0}$ and $\zeta_{k \approx 0}$ are practically independent of $g / m$ (though not of the $\Gamma$ 's which govern the rate of thermalization). This was checked varying $g / m$ including $g / m=0$, obviously a no-resonance case, up to nearly $g / m=4000$. Such results, at first surprising, may be understood looking carefully at the energies associated with the fields $\varphi_{0}, \varphi_{1}$ and $\chi$.

The graphs in Figs, 1,2 and 3 show the reheating transition for $\mathrm{g} / \mathrm{m}=3000$ and $\Gamma_{\chi}=$ $\Gamma_{\varphi}=5 \times 10^{-5}$. Fig.1 shows the energy densities of the inflaton particles $\varphi_{1}$ and of $\chi$, displaying the parametric resonance. The scale is such that in all the graphs not all the

\footnotetext{
${ }^{6}$ The suffices in and out denote the beginning and end of reheat respectively
} 
rapid oscillations are adequately displayed. Fig. 2 shows the considerable $\zeta$ oscillations during the transition and that it is rather constant in the initial period when, as we can see from Fig. 3, the density of $\varphi_{1}$ is small compared to $\varphi_{0}$. Despite considerable oscillations to larger $\zeta$ the final result for these small decay constants is that $\zeta$ decreases and by relatively little.

Although $<\chi^{2}>$ increases by many powers of ten during the resonance (more than $10^{20}$, for $g / m=3000, m=10^{-7}$, the energy associated with this field is, nevertheless, always smaller than the energy of the fields $\varphi_{0}, \varphi_{1}$, which thus control the dynamics of the system. The most striking feature is that the case $g=0$ having essentially zero $\chi$ field produces the same change in $\zeta$. This has already been found and discussed in ref. [11] for the case where there is no particle decay $\left(\Gamma_{\chi}=\Gamma_{\varphi}=0\right)$. There the analytic expression for the rate of change $\dot{\zeta}$ is non-zero due to the binary (non-perturbative) terms in the inflaton particle field, $\varphi_{1}$ even if the $\chi$ field were zero:

$$
\begin{gathered}
\frac{3}{2} H(1+w) \dot{\zeta}=\ddot{\psi}+H \dot{\psi}+2 \dot{H} \psi-(\dot{\psi}+H \psi) \frac{d}{d t} \ln \left(\rho_{h}+p_{h}\right), \\
\frac{d}{d t} \ln \left(\rho_{h}+p_{h}\right)=2 \frac{\dot{\varphi}_{0} \ddot{\varphi}_{0}+\left\langle\dot{\varphi}_{1} \ddot{\varphi}_{1}\right\rangle+\langle\dot{\chi} \ddot{\chi}\rangle+\left\langle\dot{\varphi}_{1, i} \ddot{\varphi}_{1, i}\right\rangle / 3+\left\langle\dot{\chi}_{, i} \ddot{\chi}_{, i}\right\rangle / 3}{\dot{\varphi}_{0}^{2}+\left\langle\dot{\varphi}_{1}^{2}\right\rangle+\left\langle\dot{\chi}^{2}\right\rangle+\left\langle\dot{\varphi}_{1, i} \dot{\varphi}_{1, i}\right\rangle / 3+\left\langle\dot{\chi}_{, i} \dot{\chi}_{, i}\right\rangle / 3},
\end{gathered}
$$

Thus the presence of the inflaton particles, comparable in their contribution to the energy to the residual homogeneous inflaton field, generates a change in $\zeta$ in the reheating - or more exactly the preheating - period. However as we see from Table I, for the small value of $\Gamma_{\varphi}$ chosen (for the reasons given above), the ultimate change in $\zeta$ is quite small.

\section{B. Large coupling constant, $\mathrm{g} / \mathrm{m}$}

We see in Table II that $\zeta$ increases in this regime: $\zeta_{\text {out }}>\zeta_{\text {in }}$. The graphs in Figs, 4, 5 and 6 show the reheating transition for $g / m=4700$ and $\Gamma_{\chi}=\Gamma_{\varphi}=5 \times 10^{-5}$. We may note that: firstly the parametric resonance in $\chi$ is greater as one would expect with a larger coupling; secondly that $\zeta_{\text {out }}$ now varies with $g$. Thirdly the fluctuations in $\zeta$ tend to be larger, with a considerable increase in a smoothed value of $\zeta$ before true heating takes over, the increase taking off when the $\chi$ density attains the $\varphi_{1}$ density as can be seen from Figs. 4 and 5. Again we should emphasize the role of the decay widths; if these had been much larger in that case then the final $\zeta$ would have been much changed.

\section{The sudden approximation}

It may be of interest to compare our results with those of the sudden approximation, where there is no use of the reheating mechanism, especially because of the independence of $g$ in a range of smaller couplings set out in section IIIA. The matching conditions, deduced by Deruelle and Mukhanov [20] from the Lichnerowicz conditions, imply [21] that $\psi$ and the function 


$$
\Gamma \equiv\left(\psi^{\prime} \alpha^{-1}+\psi-\frac{1}{3} \nabla^{2} \psi \alpha^{-2}\right) /\left(1-\alpha^{\prime} \alpha^{-2}\right)
$$

as well as $\alpha=a^{\prime} / a$, be continuous on the sudden transition hypersurface, say $\tau=\tau_{2}$. At the end of inflation, that is on one side, denoted $\tau_{2-}$, of the transition hypersurface, the values of $\psi$ and $\Gamma$ may be written

$$
\begin{aligned}
& \psi\left(\tau_{2-}\right)=\frac{p+1}{2 p+1} \Sigma, \\
& \Gamma\left(\tau_{2-}\right)=\frac{p}{2 p+1} \Sigma,
\end{aligned}
$$

where $\Sigma \propto y^{p}$ is given by the inflation model as in section IIB. On the other side of the hypersurface in the radiation era, we may write the general solution for $\psi$

$$
\psi_{k}=\left[B_{1}(\epsilon \cos \tilde{\epsilon}-\sin \tilde{\epsilon})+B_{2}(\cos \tilde{\epsilon}+\epsilon \sin \tilde{\epsilon})\right] / a \epsilon^{2},
$$

where $\tilde{\epsilon}=\epsilon-\epsilon_{2}, \epsilon=k \tau / \sqrt{3}$ and $\epsilon_{2}=k \tau_{2} / \sqrt{3}$ is the value of $\epsilon$ at the beginning of the radiation era. (We take the convention on $\tau$ that $a=c \tau, c$ constant, in the radiation era.) From Eq.(39) we can obtain the values of $\psi$ and $\Gamma$ on the radiation era side of the hypersurface denoted $\tau_{2+}$. Then solving

$$
\begin{gathered}
\psi\left(\tau_{2+}\right)=\psi\left(\tau_{2-}\right), \\
\Gamma\left(\tau_{2+}\right)=\Gamma\left(\tau_{2-}\right),
\end{gathered}
$$

gives

$$
\begin{gathered}
B_{1} \epsilon_{2}=-2 a_{2} \Sigma+2 \epsilon_{2}^{2} a_{2} \frac{p+1}{2 p+1} \Sigma, \\
B_{2}=2 a_{2} \Sigma-\epsilon_{2}^{2} a_{2} \frac{p+1}{2 p+1} \Sigma
\end{gathered}
$$

For values of $k$ relevant to the CMBRF we can expand $\psi$ in powers of $\epsilon$ through the pure radiation era:

$$
\begin{aligned}
& \psi(\tau)=\psi_{q a}+O\left(\epsilon_{2}^{2} / \epsilon^{2}\right) \\
& \psi_{q a}=\frac{2}{3} \frac{\epsilon}{\epsilon_{2}} \frac{a_{2}}{a} \Sigma=\frac{2}{3} \Sigma
\end{aligned}
$$

where in the last equation we have used the fact that both $a$ and $\epsilon$ are proportional to $\tau$. Thus, in the radiation era, $\psi$ changes rapidly at the beginning and after a few efolds of $a$ takes up a constant quasi-asymptotic value, $\psi_{q a}$. This constancy of $\psi$ in most of the radiation era is a general property and not peculiar to its initiation by a sudden transition. 
For the sudden transition one finds that $\psi_{q a}$ is considerably bigger than $\psi_{i n}$, the value at the end of inflation:

$$
\psi_{q a}=\frac{2}{3} \frac{2 p+1}{p+1} \psi_{i n}=7.33 \psi_{i n}
$$

The corresponding quasi-asymptotic value found in our gradual reheating calculations for $g / m<4500$ is surprisingly close:

$$
\psi_{q a} \approx 7.0 \psi_{i n}
$$

A similar explicit evaluation of $\zeta$ in the sudden transition case gives from the beginning of and all through the radiation era

$$
\zeta=\frac{\epsilon}{\epsilon_{2}} \frac{a_{2}}{a} \Sigma=\Sigma=\zeta_{\text {in }}
$$

In the gradual reheating calculations for $g / m<4500$

$$
\zeta=\zeta_{\text {out }}=.88 \zeta_{\text {in }}
$$

Thus when parametric resonance is small our gradual transition calculation and the sudden transition result approximate each other to within about $12 \%$ for $\zeta$ and $5 \%$ for $\psi$.

\section{CMBR FLUCTUATIONS; QUADRUPOLE MOMENT}

We consider that the $\zeta$ effects we have calculated for a non-linear theory would apply, qualitatively, to a large range of parameters. Nevertheless we should show that the parameters used here are quite near to ones which correctly normalise the theory to the cosmic microwave background fluctuations as required for modelling the real world.

So in this section we shall be concerned with the calculation of the scalar perturbations in the CMBR, through the consideration of the Sachs-Wolf effect, describing the fractional variation of the temperature $d T / T$. Our procedure will not involve details of the radiation to matter era transition or of the matter era itself and so will only be applicable to the lower multipoles of the radiation; in particular we shall calculate the quadrupole moment to obtain the CMBR normalization. Here we briefly describe how we implement a rather standard method (see for example ref. [26]) using the power function of $\psi$. (We have also calculated using another method, that of ref. [21], which takes account of some details and have obtained similar results.) We track the development of the metric perturbation $\psi$, from the end of reheating, through the radiation era and into the matter era.

The cosmic microwave background is described by a single number, the temperature $T$ of the black body radiation. The small anisotropies in it from the direction dependent fluctuations, $\delta T(\mathbf{e})$, where $\mathbf{e}$ is a unit vector in the direction of observation, depend on the metric perturbation along the light path after emission at last scattering - the Sachs-Wolfe effect. This can be expressed as [25,26]

$$
\frac{\delta T}{T}(\mathbf{e})=-\frac{1}{5} \mathcal{R}\left(\mathbf{x}_{L S}, \tau_{L S}\right)
$$


where $\mathbf{x}_{L S}=\frac{2}{H_{0}} \mathbf{e}, \frac{2}{H_{0}}$ being a good approximation to the distance of the last scattering sphere with $H_{0}$ the present Hubble and $\tau_{L S}$ the emission time. $\mathcal{R}\left(\mathbf{x}_{L S}, \tau_{L S}\right)$ is stochastic being given linearly by $\psi\left(\mathbf{x}_{L S}, \tau_{L S}\right)$ which is itself stochastic being expressed by the corresponding formula to Eq.(5).

Using Eq.(50) the angular correlation moments, $\mathcal{C}_{l}$, can be calculated in terms of $\mathcal{R}_{k}\left(\tau_{L S}\right)$. With ensemble averaging using Eq.(6) it can be shown, after some manipulation [26], that

$$
\mathcal{C}_{l}=\frac{4 \pi}{25} \int \frac{d k}{k} \mathcal{P}_{\mathcal{R}}(k)\left[j_{l}\left(\frac{2 k}{a_{0} H_{0}}\right)\right]^{2}
$$

where $\mathcal{P}_{\mathcal{R}}(k)$ is the power function of the curvature perturbation:

$$
\mathcal{P}(k)=\frac{k^{3}}{2 \pi^{2}}\left|\mathcal{R}_{k}\right|^{2}
$$

To calculate low moments we only need $\mathcal{R}_{k}\left(\tau_{L S}\right)$ for values of the order of $a_{0} H_{0}$ and at that time they are well outside the horizon, $k \ll a H\left(\tau_{L S}\right)$ and so $\mathcal{R}_{k} \approx-\zeta_{k}$ to a good approximation. So we can replace $\mathcal{R}$ by $\zeta$ in Eqs.(51) and (52. Knowing $\zeta_{k}$ at the end of inflation for an appropriate range of $k$ enables the calculation of correlation moments using these equations. This can be done in principle by computing $\zeta_{k}$ for sufficient $k$-values to cover the relevant range defined by the spherical Bessel functions. Alternatively we note from section [IB that $\psi_{k}, \zeta_{k} \propto k^{p-1 / 2}$ at the beginning of reheating. The values of $k / a H$ in the reheating period are extremely small for values of $k$ appropriate to the CMBRF (and indeed even much larger values) and so the $k^{2}$ terms in the reheating equations are negligable. Thus the $k$-dependence passes unaltered through the reheating and we use it to perform the $k$-integration of Eq. (51) to produce the results which now follow.

We calculate the quadrupole moment defined, with $l=2$, as

$$
Q=T_{0} \sqrt{\frac{2 l+1}{4 \pi} \mathcal{C}_{l}}
$$

Let $R_{\zeta} \equiv \zeta_{k, \text { out }} / \zeta_{k, \text { in }}$, this ratio being independent of $k$ for the relevant $k$ and is found from the numerical solution of the reheat equations (Tables I and II). In terms of the $k$-independent quantity $R_{\zeta} \zeta_{k, i n} k^{1 / 2-p}$ we find

$$
\begin{gathered}
\mathcal{C}_{2}=I_{p}\left[R_{\zeta}\left|\zeta_{k, i n}\right| k^{1 / 2-p}\left(a_{0} H_{0}\right)^{p+1}\right]^{2} \\
I_{p}=\frac{2}{25 \pi} \int \frac{d z}{z} z^{2 p+2} j_{2}(2 z) .
\end{gathered}
$$

From Eqs. 27-32

$$
\begin{gathered}
\left|\zeta_{k, \text { in }}\right| k^{1 / 2-p}\left(a_{0} H_{0}\right)^{p+1}=\sqrt{\frac{2 \pi p}{p+1}}\left(\frac{a_{0} H_{0}}{a_{\text {in }} H_{\text {in }}}\right)^{p+1} \frac{H_{\text {in }}}{m_{\text {Planck }}}|\tilde{M}(p)| \\
\tilde{M}(p)=\frac{\sqrt{\pi}(-p / 2)^{p}}{\sin ((1 / 2-p) \pi) \Gamma(p+1 / 2)} .
\end{gathered}
$$


For $g / m<4500$ and for the values of the decay constants given in Table I we find

$$
Q \approx 12 \mu K .
$$

If $\zeta$ had passed unchanged through reheating we would have found

$$
Q \approx 14 \mu K
$$

The observed value is $Q \approx 18 \mu K$, and our theoretical result can readily be changed to agree with this by adjustment of the parameters $\varphi_{0, i n}$ - the homogeneous inflaton field at the end of inflation - and $m$, the reheating potential parameter. Similar relatively small adjustments to these parameters (while scaling $g, M, \Gamma_{\chi}$ and $\Gamma_{\varphi}$ proportionately to $m$ ) can give agreement with the observed $Q$ for larger couplings such as those of Table II.

Of course, while the evaluation above verifies that the parameters we have used are in the right region, the point is not the precise values of $Q$ found but rather the amount of change induced in $\zeta$ by the reheating which forces changes in the parameters of the theory.

\section{SUMMARY AND DISCUSSION}

We have used a well-known simple theory that can give rise to parametric resonance of a scalar field, $\chi$, coupled to the scalar inflaton, $\varphi$, through a term $g^{2} \varphi^{2} \chi^{2}$. We have investigated preheating, followed by reheating mediated by decay terms $\Gamma_{\varphi} \dot{\varphi}^{2}$ and $\Gamma_{\chi} \dot{\chi}^{2}$ in the Lagrangian which implement a smooth transition to a radiation era with a hydrodynamic fluid of relativistic particles. We have arranged a transition from an inflationary era ro the heating era that is also smooth. Besides non-linear effects of the extra field $\chi$ we have included non-linear effects of the inflaton particle field $\varphi_{1}$. The investigations centre on the three-curvature related parameter $\zeta_{k}$ for values of $k$ relevant to the cosmic microwave background fluctuations. While, for such $k, \zeta_{k}$ is constant through the latter part of inflation and also through the radiation and subsequent eras as far as the generation of the fluctuations, the results presented show $\zeta_{k}$ varying in the heating transition. Thus they emerge into into the radiation era with a value changed from that in inflation which implies a change in the fluctuations - or rather a change in the parameters of the theory from what they would have been if $\zeta$ had been constant throughout. The change depends markedly on the magnitudes of $g^{2}, \Gamma_{\varphi}$ and $\Gamma_{\chi}$.

For given values of $\Gamma_{\varphi}$ and $\Gamma_{\chi}$ there is a range of $g^{2}$ from small up to some value depending on the the magnitudes of $\Gamma_{\varphi}$ and $\Gamma_{\chi}$ where parametric resonance occurs but where the change in $\zeta$ is always by the same proportion. Though in the results presented this change is a diminution only of the order of $10 \%$ or so, it is interesting that it appears not to be due to the parametric resonance in $\chi$ but only to non-linear effects of the inflaton particles produced - the energy density of the $\chi$ particles never quite reaching that of $\varphi$ particles. This change in $\zeta$ should be no great surprise as when $\left\langle\varphi_{1}^{2}\right\rangle$ is comparable with $\left|\varphi_{0}^{2}\right|$, as it is during the latter stages of heating, theorems on the constancy of $\zeta$ break down. However there is a range of $g^{2}, \Gamma_{\varphi}$ and $\Gamma_{\chi}$ where there is considerable parametric resonance but the view that $\zeta$ should be unvarying throughout is a reasonable approximation.

With larger $g^{2}$ then $\zeta$ increases by a considerable proportion this being up to a factor of 20 in the examples we have given. These cases are characterised by the $\chi$ energy density, 
after the onset of parametric resonance, approaching and at times surpassing the $\varphi_{1}$ energy density. As can be seen from the figures , $\zeta$ fluctuates markedly after 2 or 3 efolds into the heating transition. If we make the $\Gamma$ s larger so that the decay to radiation is quicker then the variability of the radiation era $\zeta$ with $g^{2}$ increases.

In the inflationary period the $\chi$ field is restricted to within about the primordial value of quantum fluctuations. As seen from Figures 1 and 3 this persists for a few efolds into the heating transition before parametric resonance sets in and raises the energy density by about 50 powers of ten in half an efold. Subsequently the $\chi$ energy density never greatly exceeds that of the $\varphi_{1}$; it seems that the direct interaction between these two fields is a strongly limiting factor. That is, the same interaction that gives rise to parametric resonance may also act to limit it because it contains inflaton particles as well as the classical inflaton field, $\varphi_{0}$. This feature seems likely to apply to many other models with parametric resonance. So then the inflaton particles always play an important role in the evolution of $\zeta$ and the revelation of their significance may be the most important result of the non-linear theory.

The formalism we use has been explained at much greater length in a preceeding paper [10]. In that paper also fairly strong $\zeta$ variations were computed but the results given here supercede (see Section [II] those previous ones [10,11. Also the present work extends the previous work by treating the completion of reheating into the radiation era where $\zeta$ becomes a constant, different from that at the end of inflation, and thus the consequences for the cosmic microwave background fluctuations become evident. Our model extends smoothly from the primordial quantum excitations to the creation of the fluctuations.

The main feature of our formalism is that as the number density of the original quantum fields increases a smooth transition to where they can be treated as classical stochastic occurs 18]; the only manageable treatment of the non-linear terms in the equations of motion is to take ensemble averages. This gives rise to loop imtegral factors, such as that of Eq.(1), in the equations of motion. The evaluation of these involves both an infra-red, low $k$, cut-off and an ultra-violet, high $k$, cut-off. This has been discussed in detail in Appendix B of ref. [10] where the low $k$ cut-off was regarded as a contribution to the renormalization of the homogeneous part of the field. The high $k$ cut-off is fixed to correspond to a wavelenth $H^{-1}$, the inverse Hubble length at the beginning of heating. Such a cut-off has been used in the work of other authors for example in ref. [12]. Though the only obvious approximate magnitude it is a major source of uncertainty in our work, as any cut-off very much less or very much greater would significantly affect our results. A very much smaller cut-off of course corrsponds cloosely to the neglect of all non-linear effects.

Where, on the contrary, we have used a linear approximation is in working in the equations of motion to first order in the metric perturbation $\psi$. The measure of validity we have taken is, in accord with the metric Eq.(1), to compare $2 \sqrt{<\psi^{2}>}$ with unity [10]. In cases where $\zeta$ decreases this corresponds throughout the heating process to a less than $1 \%$ perturbation to the metric and the first order perturbation theory is well justified. But in cases where $\zeta$ increases it can reach up to nearly a $10 \%$ perturbation in the latter stages of reheating. While we do not think that this gives doubt that these are cases where $\zeta$ increases markedly nevertheless the quantitative aspect would benefit from a more refined treatment.

We find it difficult to see any direct observational test of the changes in the heating transition. In the body of the paper we have used that the shape of the power spectrum, in our theory a power law, emerges unchanged from the transition and so only depends on the 
inflationary theory. The parameters of nearly all envisaged theories can be changed to follow the magnification (or diminution) of $\zeta$ and adjust to the COBE or other normalization. The dependence on the details of preheating and reheating makes this adjustment uncertain in the present state of knowledge. The best chance may lie in a comparative calculation of gravitational wave production in the hope that this can be observed [28,22]. 


\section{REFERENCES}

[1] J. H. Traschen and R. H. Brandenberger, Phys. Rev. D42 (1990) 2491 ;Y. Shtanov, J. H. Traschen and R. H. Brandenberger, Phys. Rev. D51 (1995) 5438.

[2] L. A. Kofman, A. Linde and A. A. Starobinsky, Phys. Rev. Lett. 73 (1994) 3195.

[3] D. Boyanovsky, H. J. de Vega, R. Holman, D.-S. Lee and A. Singh Phys. Rev. D51 (1995) 4419;M. Yoshimura, Prog. Theor. Phys. 94 (1995) 8873;D. Kaiser, Phys. Rev. D53 (1996) 1776; H. Fujisaki, K. Kumekawa, M. Yamaguchi and M. Yoshimura, Phys. Rev. D53, 6805 (1996); J. Baacke, K. Heitmann and C. Patzold, Phys. Rev. D56, 6556 (1997).

[4] L. A. Kofman, A. Linde and A. A. Starobinsky, Phys. Rev. D 56, 3258, (1997).

[5] L.A.Kofman,In:Relativistic Astrophysics: A Conference in Honor of Igor Novikof's 60th Birthday. Copenhagen 1996,Eds. B. Jones and D. Markovitch, Cambridge University Press; also at astro-ph/9605155.

[6] S. Yu. Khlebnikov and I. I. Tkachev, Phys. Rev. Lett. 77, 219 (1996); Phys. Lett. B 390, 80 (1997); Phys. Rev. Lett. 79, 1607 (1997).

[7] D. Boyanovsky, D. Cormier, H. J. de Vega, R. Holman and S. P. Kumar, Proc. VIth Erice Chalonge School on Astrofundamental Physics, Ed.N. S. Vanchez and A. Zichichi (Kluwer,1998) hep-ph/9801453 and references therein.

[8] F. Finelli and R. Brandenberger, Phys. Rev. Lett. 82, (1999) 1362 , hep-ph/9809490.

[9] F. Finelli and R. Brandenberger, Phys. Rev. D62 (2000) 083502.

[10] A. B. Henriques and R. G. Moorhouse, Phys. Rev. D62, 063512-1 (2000), hepph/0003141.

[11] A. B. Henriques and R. G. Moorhouse, Phys. Lett. B492, 331, (2000)

[12] A. R. Liddle. D. H. Lyth, K. A. Malik and D. Wands, Phys. Rev. D61 (2000) 103508; hep-ph/9912473.

[13] B. A. Bassett, D. I. Kaiser, F. Tamburini and R. Maartens, Nucl. Phys.B 561 (1999),188; hep-ph/9901319v3.

[14] K. Jedamzik and G. Sigl, Phys. Rev.D61 (2000) 023519; hep-ph/9906287.

[15] J. M. Bardeen,P. J. Steinhardt and M. S. Turner, Phys. Rev. D 28, 679, (1983).

[16] D. H. Lyth, Phys. Rev. D31, 1792 (1985).

[17] V. F. Mukhanov, H. A. Feldman and R. H. Brandenburger, Phys. Rep. 215, 203 (1992).

[18] D. Polarski and A. A. Starobinsky, Class. Quant. Grav., 13, 377 (1996).

[19] F. Finelli and S. Khlebnikov, hep-ph/0107143

[20] N. Deruelle and V. F. Mukhanov, Phys. Rev. D52, 5549 (1995).

[21] A. B. Henriques, L. E. Mendes and R. G. Moorhouse, Phys. Rev. D 55,5908,1997.

[22] L. P. Grishchuk, Phys. Rev. D50, 7154 (1994).

[23] J. Garcia-Bellido and D. Wands, Phys. Rev. D52, 6739 (1995)..

[24] D. Wands, K. A. Malik, D. H. Lyth and A. R. Liddle, astro-ph/0003278.

[25] R. Sachs and A. Wolfe, Astrophys. J. 147, 73 (1967).

[26] A. R. Liddle and D. H. Lyth. 'Cosmological Inflation and Large Scale Structure', Cambridge University Press, 2000 (ISBN 0-521-66022-X).

[27] N. Deruelle and V. F. Mukhanov, Phys. Rev. D52, 5549 (1995).

[28] S. Yu. Khlebnikov and I. I. Tkachev, Phys. Rev. D 56, 653, (1997).

[29] Bruce A. Bassett,Phys. Rev. D 56, 3439, (1997). 


\section{TABLES}

TABLE I. The change in $\zeta$ in the inflation to radiation era transition, shown for smaller values of the $\chi, \varphi$ coupling, $g$. Also shown are the increase, $n$ (efolds), in $\ln (a)$ in the transition and the ratio of densities of $\chi$ and $\varphi_{1}$ at the maximum density of $\chi$. The decay constants are given by $\Gamma_{\chi} / m=\Gamma_{\varphi} / m=5 \times 10^{-5}$.

\begin{tabular}{|c|c|c|c|}
\hline \hline$g / m$ & $n($ efolds $)$ & $\log \left(\operatorname{den} \chi /\right.$ den $\left.\varphi_{1}\right)$ & $\zeta_{\text {out }} / \zeta_{\text {in }}$ \\
\hline 1000 & 7.9 & -39 & 0.88 \\
3000 & 7.6 & -3.9 & 0.87 \\
4000 & 7.9 & -14 & 0.88 \\
\hline \hline
\end{tabular}

TABLE II. The increase of $\zeta$ in the inflation to radiation era transition, shown for some larger values of the $\chi, \varphi$ coupling, $g$. Also shown are the increase, $n$ (efolds), in $\ln (a)$ in the transition and the ratio of densities of $\chi$ and $\varphi_{1}$ at the maximum density of $\chi$. The decay constants are given by $\Gamma_{\chi} / m=\Gamma_{\varphi} / m=5 \times 10^{-5}$.

\begin{tabular}{|c|c|c|c|}
\hline \hline$g / m$ & $n($ efolds $)$ & $\log \left(\operatorname{den} \chi / \operatorname{den} \varphi_{1}\right)$ & $\zeta_{\text {out }} / \zeta_{\text {in }}$ \\
\hline 4700 & 7.9 & -.9 & 5.6 \\
4900 & 7.9 & -.9 & 10.2 \\
5000 & 7.6 & -.5 & 19.1 \\
7000 & 7.5 & +.7 & 6.3 \\
12000 & 7.9 & -.9 & 10.3 \\
\hline \hline
\end{tabular}




\section{FIGURES}

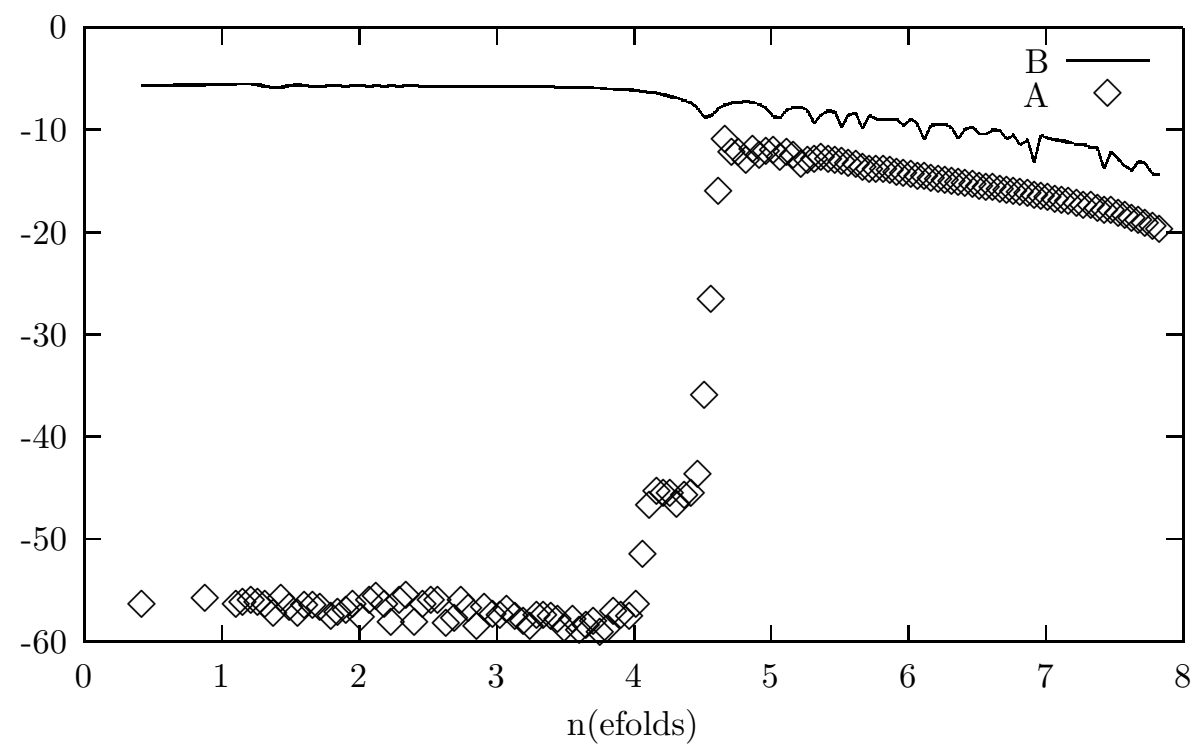

FIG. 1. For $g / m=3000$, logarithmic plot of energy densities of $\chi(\mathrm{A})$ and $\varphi_{1}$ (B) versus the number of e-folds of expansion in the reheat era. In all figures densities are in units $m^{2} \times m_{\text {Planck }}^{2}$, where $m=10^{-7} m_{\text {Planck. }}$.

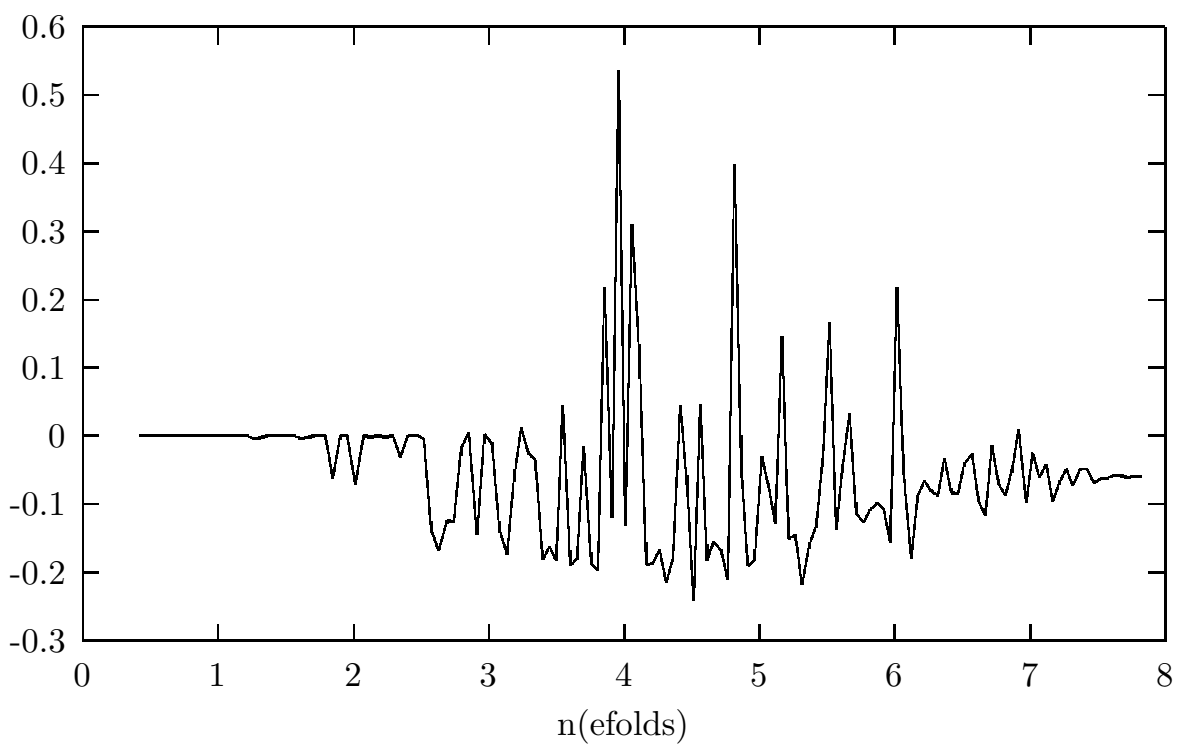

FIG. 2. For $g / m=3000$, plot of $\lg \left(\zeta / \zeta_{\text {in }}\right)$ versus the number of e-folds of expansion in the reheat era. 


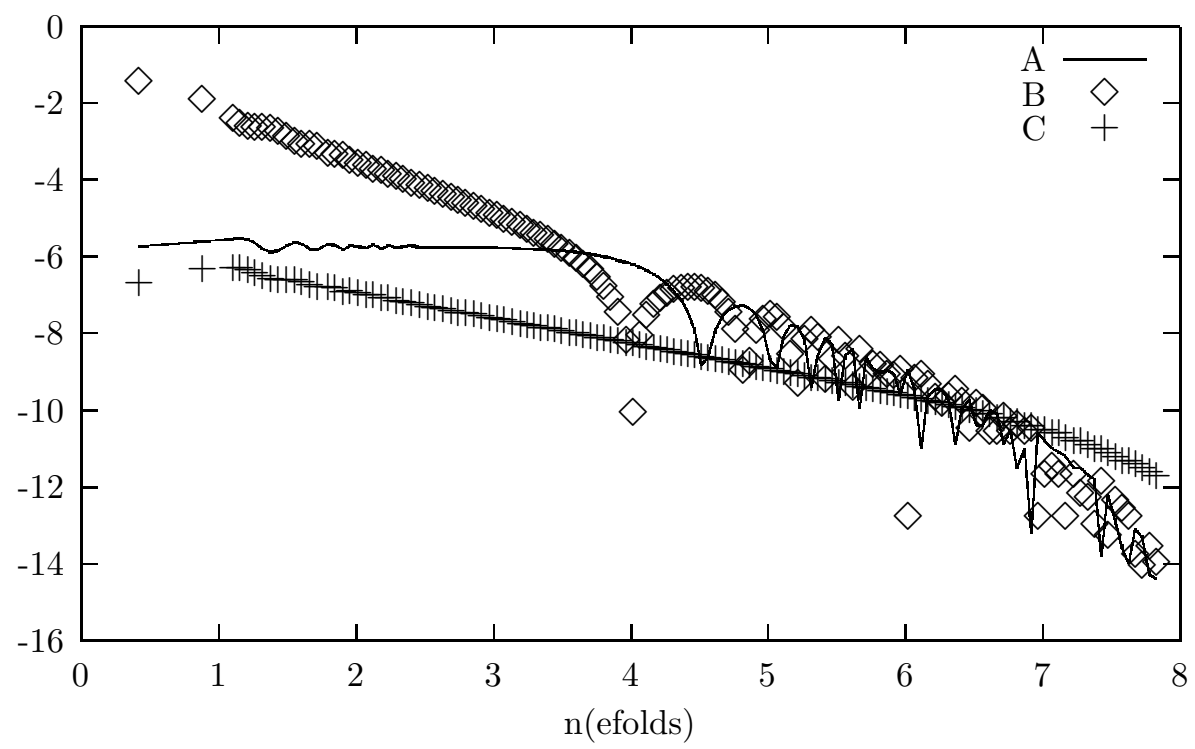

FIG. 3. For $g / m=3000$, logarithmic plot of energy densities of $\varphi_{1}(\mathrm{~A}), \varphi_{0}(\mathrm{~B})$ and $\rho_{0}$ versus the number of e-folds of expansion in the reheat era.

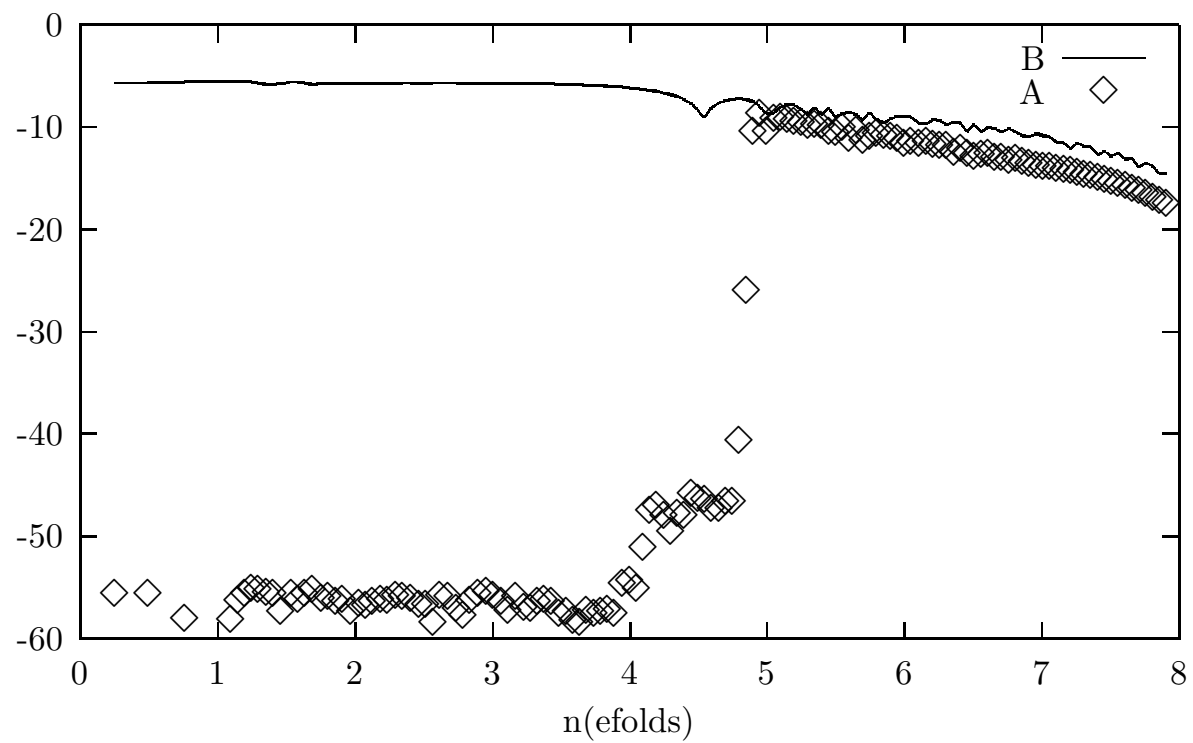

FIG. 4. For $g / m=4700$, logarithmic plot of energy densities of $\chi(\mathrm{A})$ and $\varphi_{1}$ (B) versus the number of e-folds of expansion in the reheat era. 


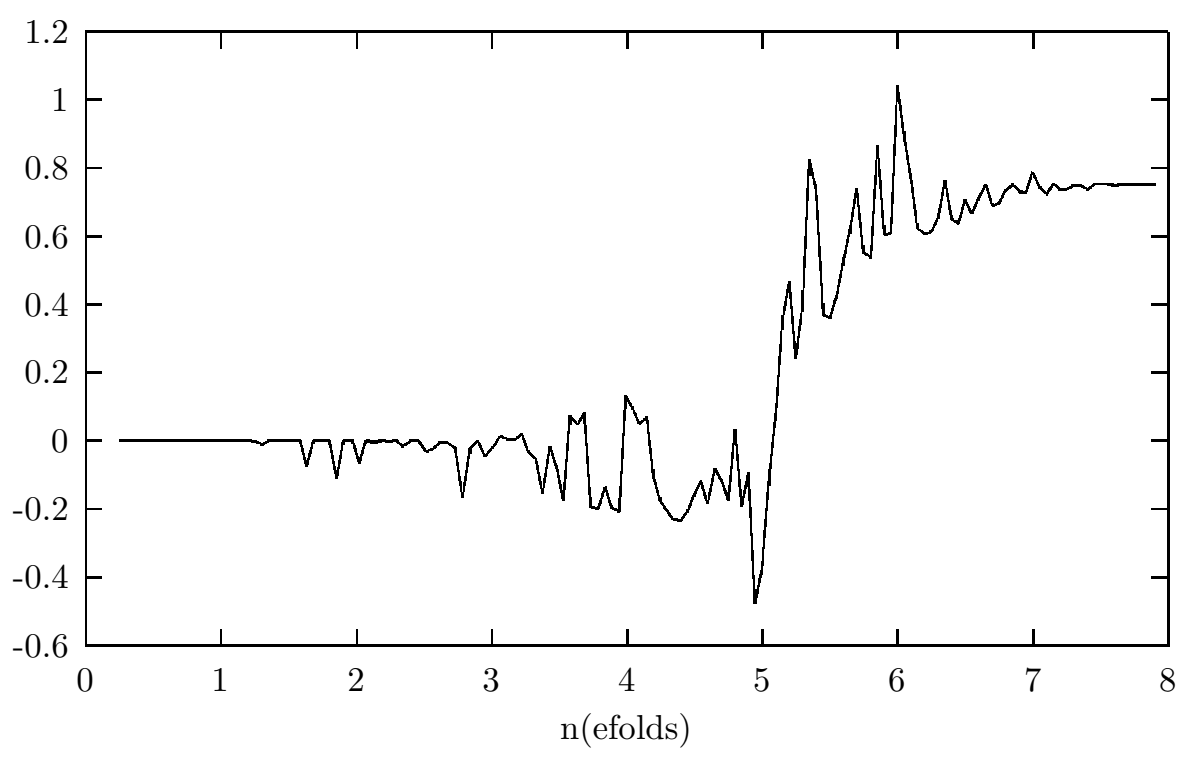

FIG. 5. For $g / m=4700$, plot of $\lg \left(\zeta / \zeta_{\text {in }}\right)$ versus the number of e-folds of expansion in the reheat era.

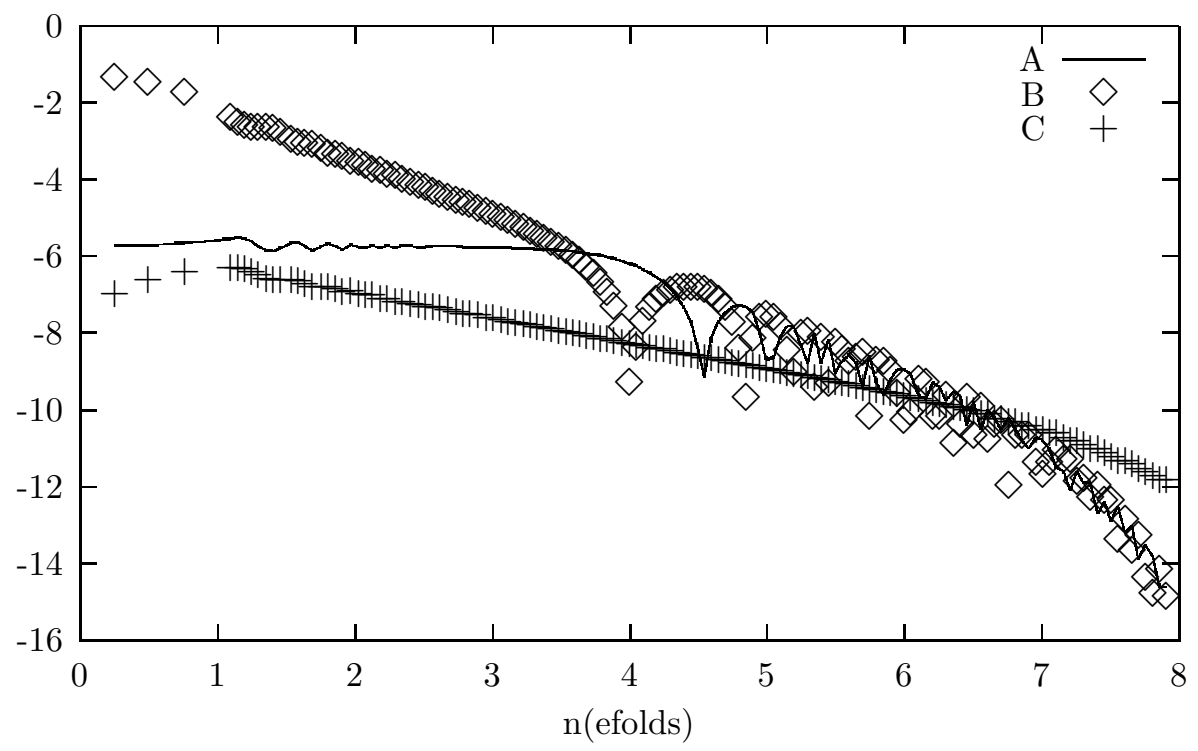

FIG. 6. For $g / m=4700$, logarithmic plot of energy densities of $\varphi_{1}(\mathrm{~A}), \varphi_{0}(\mathrm{~B})$ and $\rho_{0}$ versus the number of e-folds of expansion in the reheat era. 